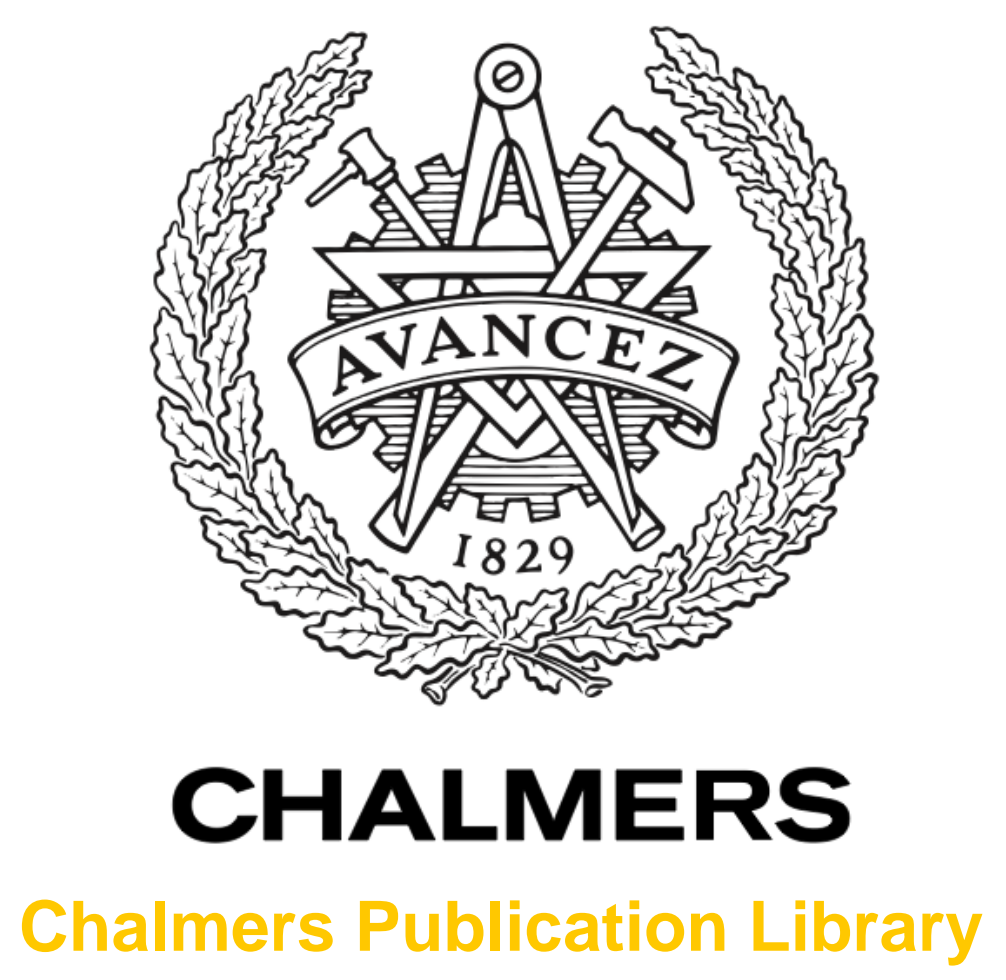

Interface damage modeled by spring boundary conditions for in-plane elastic waves

This document has been downloaded from Chalmers Publication Library (CPL). It is the author's version of a work that was accepted for publication in:

Wave motion (ISSN: 0165-2125)

Citation for the published paper:

Golub, M. ; Boström, A. (2011) "Interface damage modeled by spring boundary conditions for in-plane elastic waves". Wave motion, vol. 48(2), pp. 105-115.

http://dx.doi.org/10.1016/j.wavemoti.2010.09.003

Downloaded from: http://publications.lib.chalmers.se/publication/138450

Notice: Changes introduced as a result of publishing processes such as copy-editing and formatting may not be reflected in this document. For a definitive version of this work, please refer to the published source. Please note that access to the published version might require a subscription.

Chalmers Publication Library (CPL) offers the possibility of retrieving research publications produced at Chalmers University of Technology. It covers all types of publications: articles, dissertations, licentiate theses, masters theses, conference papers, reports etc. Since 2006 it is the official tool for Chalmers official publication statistics. To ensure that Chalmers research results are disseminated as widely as possible, an Open Access Policy has been adopted.

The CPL service is administrated and maintained by Chalmers Library. 


\title{
Interface damage modeled by spring boundary conditions for in-plane elastic waves
}

\author{
Mikhail V. Golub ${ }^{\mathrm{a}, *}$, Anders Boström ${ }^{\mathrm{b}}$ \\ anstitute for Mathematics, Mechanics and Informatics, Kuban State University, Krasnodar, 350040 Russia \\ ${ }^{b}$ Department of Applied Mechanics, Chalmers University of Technology, SE-412 96 Göteborg, Sweden
}

\begin{abstract}
In-plane elastic wave propagation in the presence of a damaged interface is investigated. The damage is modeled as a distribution of small cracks and this is transformed into a spring boundary condition. First the scattering by a single interface crack is determined explicitly in the low frequency limit for the case of a plane wave normally incident to the interface. The transmission at an interface with a random distribution of small cracks is then determined and is compared to periodically distributed cracks. The cracked interface is then described by a distributed spring boundary condition. As an illustration the dispersion relation of the first modes in a thick plate with a damaged interface in the middle is given.
\end{abstract}

Keywords: elastic waves, spring boundary condition, layered composite, crack, diffraction, boundary integral equation method, delamination, cracks distribution

\section{Introduction}

Due to their intrinsic heterogeneity composite materials may be exposed to different types of defects and damage such as voids, micro-cracking, debonding between different phases etc. This may be induced by processing, fatigue, environmental conditions, diffusion debonding etc. Damage at an interface in a composite may lead to total debonding, but may also occur in the form of micro-cracks or similar. It is not obvious how to model such damage for the purpose of ultrasonic wave propagation and detection. Different approaches that seem natural include a set of micro-cracks, a thin visco-elastic layer, or a spring boundary condition. The model of damage delamination given by spring boundary conditions is more general than just a crack. Compared to multiple cracks, spring boundary conditions are more efficient for modeling of finite heterogeneous fractures [1, 2] (experimentally [3]). Baik and Thompson [4] use a quasi-static approximation to simulate an imperfect interface by a spring with mass distributed along the interface. In a different manner Rokhlin and Wang [5] and Rokhlin and Huang [6] derive very similar asymptotic boundary conditions for interface imperfections modeled by an interfacial multiphase.

\footnotetext{
${ }^{*}$ Corresponding author

Email addresses: m_golub@inbox.ru (Mikhail V. Golub), anders.bostrom@chalmers.se (Anders Boström)
} 
Many studies on the propagation of plane ultrasonic waves through an interface with a distribution of cracks, inclusions or cavities have been performed. Angel and Achenbach [7], Mikata and Achenbach [8], and Mikata [9] consider the case of a periodic array of coplanar and inclined strip-like cracks distributed over a plane and show rather small variations in transmission coefficients. Three-dimensional problems have been also investigated: a layerlike region of distributed micro-cracks in a bulk material by Achenbach and Zhang [10], a layer of inhomogeneities (cracks, spherical cavities and inclusions) are analyzed via an integral equation method by Achenbach et al [11]. In contrast to most of studies (e.g. $[4,12]$ dealing with 3D problems) where delamination is modeled as a distribution of cracks, Boström and Wickham [13] consider identical half-spaces with a distribution of contact spots on the interface between them, in order to model partly closed cracks.

The analysis performed in all these investigations show a reasonable comparison between different approaches: the transmission coefficients for the different distributions are quite similar if the crack densities are the same. This makes it reasonable to exploit the simple spring boundary conditions which needs solely knowledge of the spring stiffness.

The model presented here is a natural continuation of the work started in Boström and Golub [14] on SH wave propagation in a damaged layered waveguide, where interface damage is substituted by a spring boundary condition with spring stiffness expressed in terms of a damage parameter. This model is now extended to the case of in-plane $\mathrm{P}$ and SV waves. At first a single interface crack between two half-spaces is considered for normal incidence of a plane longitudinal or transverse wave. The solution is obtained using a type of analytical boundary integral equation method $[15,16]$. Then the reflection and transmission coefficients for normal incidence for a random and a periodic distribution of equally sized cracks at the interface between two half-spaces are calculated. At low frequencies these two situations give quite similar results, and this motivates the use of the simpler explicit expressions for the random distribution. The transmission coefficients are then transformed into a spring boundary condition by comparing with the transmission coefficient for this case. It then happens that the normal and tangential spring constants are the same, leading to a scalar spring constant. As an illustration of the influence of damage the dispersion curves of the modes in a thick two-layered plate are given.

\section{Single interface strip-like crack}

Consider first 2D in-plane waves in two elastic isotropic half-spaces with a single interface strip-like crack of width $2 l$. A coordinate $x z$ system is introduced according to Figure 1. A fixed angular frequency $\omega$ is assumed and the factor $\exp (-i \omega t)$ is suppressed. The displacement vector is denoted $\boldsymbol{u}^{j}=\left\{u_{x}^{j}, u_{z}^{j}\right\}$, where superscript $j=1$ corresponds to the lower half-space $(z<0)$ and $j=2$ to the upper half-space $(z>0)$. The material properties are determined by the Lamé constants $\lambda^{j}$ and $\mu^{j}$ and densities $\rho^{j}$. Introduce also $c_{11}^{j}=\lambda^{j}+2 \mu^{j}$. Wave motion in this case is governed by the Lamé equation

$$
c_{11}^{j} \nabla \nabla \cdot \boldsymbol{u}^{j}-\mu^{j} \nabla \times\left(\nabla \times \boldsymbol{u}^{j}\right)+\rho^{j} \omega^{2} \boldsymbol{u}^{j}=0, \quad j=1,2 .
$$


The longitudinal or P wave velocity $v_{1}^{j}$ and the transverse or SV wave velocity $v_{2}^{j}$ are

$$
\begin{gathered}
v_{1}^{j}=\sqrt{c_{11}^{j} / \rho^{j}}, \\
v_{2}^{j}=\sqrt{\mu^{j} / \rho^{j}} .
\end{gathered}
$$

The corresponding wave numbers are $k_{1}^{j}$ and $k_{2}^{j}$. The stress components are given by Hooke's law:

$$
\begin{aligned}
& \sigma_{x z}^{j}=\mu^{j}\left(\frac{\partial u_{x}^{j}}{\partial z}+\frac{\partial u_{z}^{j}}{\partial x}\right), \\
& \sigma_{z z}^{j}=\lambda^{j} \frac{\partial u_{x}^{j}}{\partial x}+c_{11}^{j} \frac{\partial u_{z}^{j}}{\partial z}, \\
& \sigma_{x x}^{j}=c_{11}^{j} \frac{\partial u_{x}^{j}}{\partial x}+\lambda^{j} \frac{\partial u_{z}^{j}}{\partial z} .
\end{aligned}
$$

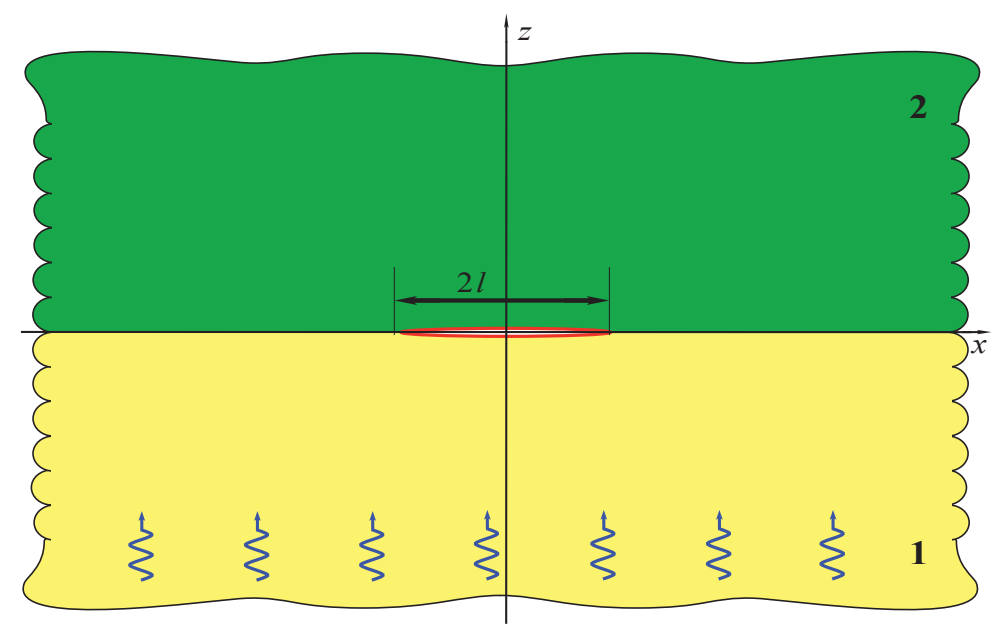

Figure 1: Geometry of the problem for a single interface crack.

Consider a plane wave incident normally on the plane interface $z=0$ containing the crack (Figure 1 ). This wave is reflected and transmitted at the interface and is scattered by the crack. The type of incident wave is specified by the index $s$ : for the P wave case $s=1$, whereas for the SV wave $s=2$. The total displacement field $\boldsymbol{u}$ is the superposition of the field $\boldsymbol{u}^{\text {in }}$ in the absence of the crack and the field $\boldsymbol{u}^{\text {sc }}$ scattered by the crack. The field in the absence of the crack is

$$
\boldsymbol{u}^{\text {in }}= \begin{cases}\boldsymbol{p}^{s}\left(\mathrm{e}^{\mathrm{i} k_{s}^{1} z}+R_{s}^{-} \mathrm{e}^{-\mathrm{i} k_{s}^{1} z}\right), & z<0, \\ \boldsymbol{p}^{s} T_{s}^{-} \mathrm{e}^{\mathrm{i} k_{1}^{2} z}, & z>0,\end{cases}
$$

where the reflection and transmission coefficients are

$$
\begin{aligned}
& R_{s}^{-}=\frac{c_{s}^{1} k_{s}^{1}-c_{s}^{2} k_{s}^{2}}{c_{s}^{1} k_{s}^{1}+c_{s}^{2} k_{s}^{2}}, \\
& T_{s}^{-}=\frac{2 c_{s}^{1} k_{s}^{1}}{c_{s}^{1} k_{s}^{1}+c_{s}^{2} k_{s}^{2}},
\end{aligned}
$$


where $s=1,2$ and the stiffness constants are $c_{1}^{j}=c_{11}^{j}$ and $c_{2}^{j}=\mu^{j}$. The subscript $s$ is omitted on most quantities in the following but this should cause no confusion. For convenience the polarization vector $\boldsymbol{p}^{s}$ describing the type of incident plane wave is used: for the $\mathrm{P}$ wave case $\boldsymbol{p}^{1}=\{0,1\}$, for the SV wave case $\boldsymbol{p}^{2}=\{1,0\}$.

The field scattered by the crack has continuous stresses $\tau^{\mathrm{sc}}=\left\{\sigma_{x z}, \sigma_{z z}\right\}$ on the interface $z=0$ while the displacement field $\boldsymbol{u}^{\text {sc }}$ has a discontinuity:

$$
\begin{cases}\boldsymbol{u}^{1, \mathrm{sc}}=\boldsymbol{u}^{2, \mathrm{sc}}, & |x|>l, \\ \tau^{1, \mathrm{sc}}=\tau^{2, \mathrm{sc}}, & |x|>l, \\ \tau^{1, \mathrm{sc}}=\tau^{2, \mathrm{sc}}=-\tau^{1, \text { in }}, & |x|<l .\end{cases}
$$

The scattered field can be represented as Fourier integrals

$$
\boldsymbol{u}^{\mathrm{sc}}=\frac{1}{2 \pi}\left\{\begin{array}{l}
\int_{-\infty}^{\infty} K^{1}(\alpha, z) \mathbf{Q}(\alpha) \mathrm{e}^{-\mathrm{i} \alpha x} \mathrm{~d} \alpha, \quad z<0, \\
\int_{-\infty}^{\infty} K^{2}(\alpha, z) \mathbf{Q}(\alpha) \mathrm{e}^{-\mathrm{i} \alpha x} \mathrm{~d} \alpha, \quad z>0,
\end{array}\right.
$$

where the Fourier transform $\left(\mathcal{F}_{x}\right)$ of the stresses at the interface appears: $\mathbf{Q}(\alpha)=\mathcal{F}_{x}\left[\tau^{\mathrm{sc}}(x, 0)\right]$. A detailed description of the derivation of Green's matrices for the 3D case has been given in $[17,16]$. In the problem under consideration only the 2D Green's matrix is used

$$
K^{j}(\alpha, z)=\frac{1}{\Delta_{j}}\left(\begin{array}{cc}
(-1)^{j} \sigma_{2, j}\left(-\alpha^{2} \mathrm{e}^{-\sigma_{1, j}|z|}+\gamma_{j}^{2} \mathrm{e}^{-\sigma_{2, j}|z|}\right) & -\mathrm{i} \alpha\left(-\gamma_{j}^{2} \mathrm{e}^{-\sigma_{1, j}|z|}+\sigma_{1, j} \sigma_{2, j} \mathrm{e}^{-\sigma_{2, j}|z|}\right) \\
-\mathrm{i} \alpha\left(-\sigma_{1, j} \sigma_{2, j} \mathrm{e}^{-\sigma_{1, j|j|}}+\gamma_{j}^{2} \mathrm{e}^{-\sigma_{2, j \mid}|z|}\right) & (-1)^{j} \sigma_{1, j}\left(-\gamma_{j}^{2} \mathrm{e}^{-\sigma_{1, j}|z|}+\alpha^{2} \mathrm{e}^{-\sigma_{2, j}|z|}\right)
\end{array}\right),
$$

where

$$
\begin{gathered}
\Delta_{j}=2 \mu^{j}\left(-\gamma_{j}^{4}+\alpha^{2} \sigma_{1, j} \sigma_{2, j}\right), \\
\sigma_{i, j}=\sqrt{\alpha^{2}-\left(\omega / v_{i}^{j}\right)^{2}}, \quad \gamma_{j}^{2}=\left(\alpha^{2}+\sigma_{2, j}^{2}\right) / 2,
\end{gathered}
$$

and the square roots are chosen according to $\operatorname{Re} \sigma_{i, j} \geq 0$ and $\operatorname{Im} \sigma_{i, j} \leq 0$.

In view of the boundary conditions (3) the Fourier transform of the stresses on the interface

$$
\mathbf{Q}(\alpha)=L(\alpha) \boldsymbol{V}(\alpha)
$$

are connected with the Fourier transform of the unknown crack-opening displacement $\boldsymbol{V}=\mathcal{F}_{x}[\boldsymbol{v}]$

$$
\boldsymbol{v}(x)=\boldsymbol{u}^{1, \mathrm{sc}}\left(x, 0^{-}\right)-\boldsymbol{u}^{2, \mathrm{sc}}\left(x, 0^{+}\right)
$$

by means of the matrix

$$
L(\alpha)=\left[K^{1}(\alpha, 0)-K^{2}(\alpha, 0)\right]^{-1} .
$$

Substitution of the integral representation for $\tau^{\mathrm{sc}}$ into (3) gives

$$
\frac{1}{2 \pi} \int_{-\infty}^{\infty} L(\alpha) \boldsymbol{V}(\alpha) \mathrm{e}^{\mathrm{i} \alpha x} \mathrm{~d} \alpha=-\mathrm{i} c_{s}^{1} k_{s}^{1}\left(1-R_{s}^{-}\right) \boldsymbol{p}^{s}
$$


This is an integral equation for the unknown crack-opening displacement.

To discretize the integral equation the crack-opening displacement is expanded in a series

$$
\boldsymbol{v}(x)=\sum_{n=1}^{\infty} \boldsymbol{\alpha}_{n} \psi_{n}(x / l)
$$

where the Chebyshev functions are used as basis functions

$$
\psi_{n}(s)=\frac{\sin (n \arccos s)}{\sin s} .
$$

These functions form a complete set on the interval $[-l, l]$ and they have a square root behaviour at the crack edges. However, it is known that the correct singularity at the crack edge also contains an oscillatory factor and this can be included in the expansion by taking Jacobi polynomials instead of Chebyshev polynomials (see [18]). These oscillations are not included here because this complicates the calculations of some integrals below and presumably the oscillations are not very important. Inserting this expansion into the integral equation Eq. (4) and projecting on the Chebyshev functions gives the following discretized form of the integral equation

$$
\sum_{n=1}^{\infty} Q_{n n^{\prime}} \boldsymbol{\alpha}_{n^{\prime}}=-\mathrm{i} H_{s} l \boldsymbol{p}^{s} \delta_{n 1},
$$

where $\delta_{n 1}$ is the Kronecker delta. The constants $H_{s}$ are defined as

$$
H_{s}=\frac{c_{s}^{1} c_{s}^{2} k_{s}^{1} k_{s}^{2}}{c_{s}^{1} k_{s}^{1}+c_{s}^{2} k_{s}^{2}}
$$

and the matrix on the left-hand side of the equation is

$$
Q_{n n^{\prime}}=\frac{1}{2 \pi} \int_{-\infty}^{\infty} L(\alpha) \mathrm{J}_{n}(\alpha l) \mathrm{J}_{n^{\prime}}(\alpha l) \frac{\mathrm{d} \alpha}{\alpha^{2}} .
$$

\section{Asymptotic solution for a single interface strip-like crack}

The procedure described above is suitable for numerical calculations. But if the crack is small an asymptotic analysis can be performed to yield an analytical expression and this is much more useful in the present case. At low frequencies $\left(\omega l / v_{i}^{j}<<1\right)$ the square roots can be expanded as

$$
\sigma_{i, j}=\alpha-\omega^{2}\left(v_{i}^{j}\right)^{-2} /(2 \alpha)
$$

This leads to the following approximation for the kernel of the integral equation (4)

$$
L(\alpha) \sim \widetilde{L} \alpha=\left[\widetilde{K}^{1}-\widetilde{K}^{2}\right]^{-1} \alpha
$$

where

$$
\widetilde{K}_{j}=-\frac{1}{2 \mu^{j}\left(\lambda^{j}+\mu^{j}\right)}\left(\begin{array}{cc}
(-1)^{j} c_{11}^{j} & \mathrm{i} \mu^{j} \\
-\mathrm{i} \mu^{j} & (-1)^{j} c_{11}^{j}
\end{array}\right) .
$$


Subsequently, the asymptotic approximation of the kernel becomes

$$
\widetilde{L}=\frac{2}{\beta_{1}^{2}-\beta_{2}^{2}}\left(\begin{array}{cc}
\beta_{1} & -\mathrm{i} \beta_{2} \\
\mathrm{i} \beta_{2} & \beta_{1}
\end{array}\right)
$$

which only depends on the elastic constants

$$
\beta_{1}=\frac{c_{11}^{1}}{\left(\lambda^{1}+\mu^{1}\right) \mu^{1}}+\frac{c_{11}^{2}}{\left(\lambda^{2}+\mu^{2}\right) \mu^{2}}, \quad \beta_{2}=\frac{1}{\lambda^{1}+\mu^{1}}-\frac{1}{\lambda^{2}+\mu^{2}} .
$$

With this low frequency approximation the matrix in the system of equations can be calculated analytically:

$$
Q_{n n^{\prime}}=\frac{\widetilde{L}}{2 \pi} \int_{-\infty}^{\infty} \mathrm{J}_{n}(\alpha l) \mathrm{J}_{n^{\prime}}(\alpha l) \frac{\mathrm{d} \alpha}{\alpha}=\frac{\widetilde{L}}{2 \pi n} \delta_{n n^{\prime}} .
$$

The crack-opening displacement for an incident $\mathrm{P}$ wave at low frequencies then becomes

$$
\boldsymbol{v}_{0}^{L}(x)=\mathrm{i} H_{L}\left(\begin{array}{c}
\mathrm{i} \beta_{2} \\
\beta_{1}
\end{array}\right) \sqrt{l^{2}-x^{2}} .
$$

For an incoming SV wave a very similar expression is obtained

$$
v_{0}^{T}(x)=\mathrm{i} H_{T}\left(\begin{array}{c}
\beta_{1} \\
-\mathrm{i} \beta_{2}
\end{array}\right) \sqrt{l^{2}-x^{2}}
$$

In this case with different materials in the two half-spaces the crack-opening displacement has two components in general. When the two materials are the same, or more generally when $\lambda^{1}+\mu^{1}=\lambda^{2}+\mu^{2}, \beta_{2}=0$ and there is only one component.

To estimate the accuracy of the asymptotic crack-opening displacement, the exact average computed from (5) is compared to the average calculated from the asymptotic formulae (6) or (7). The average value of the crack opening displacement is defined as

$$
\overline{\mathbf{v}}=\frac{1}{2 l} \int_{-l}^{l} \boldsymbol{v}(x) d x .
$$

The ratio between the asymptotic low frequency solution and the exact solution for an incident $\mathrm{P}$ wave $\bar{v}_{z}^{L} / \bar{v}_{0 z}^{L}$ is shown in Figure 2, with the real and imaginary parts shown separately. The densities in the two half-spaces are assumed to be equal $\rho^{1}=\rho^{2}$, while four different ratios between the elastic constants are considered: $c_{i j}^{2}=B c_{i j}^{1}, B=1,2,3,4$, where $c_{i j}$ is any of the elastic constants. This implies that the Poisson ratios $\nu^{j}=\lambda^{j} \cdot\left[2\left(\lambda^{j}+\mu^{j}\right)\right]^{-1}$ are also equal, and they are chosen as $v^{1}=v^{2}=0.3333$. The low frequency asymptotic solution is accurate within a few percent up to dimensionless frequency around 0.3. As the focus here is on small interface cracks due to damage, the asymptotic solution is used in the following. This also has the great advantage of giving explicit formulae for the crack-opening displacement and later also for the spring constant. 

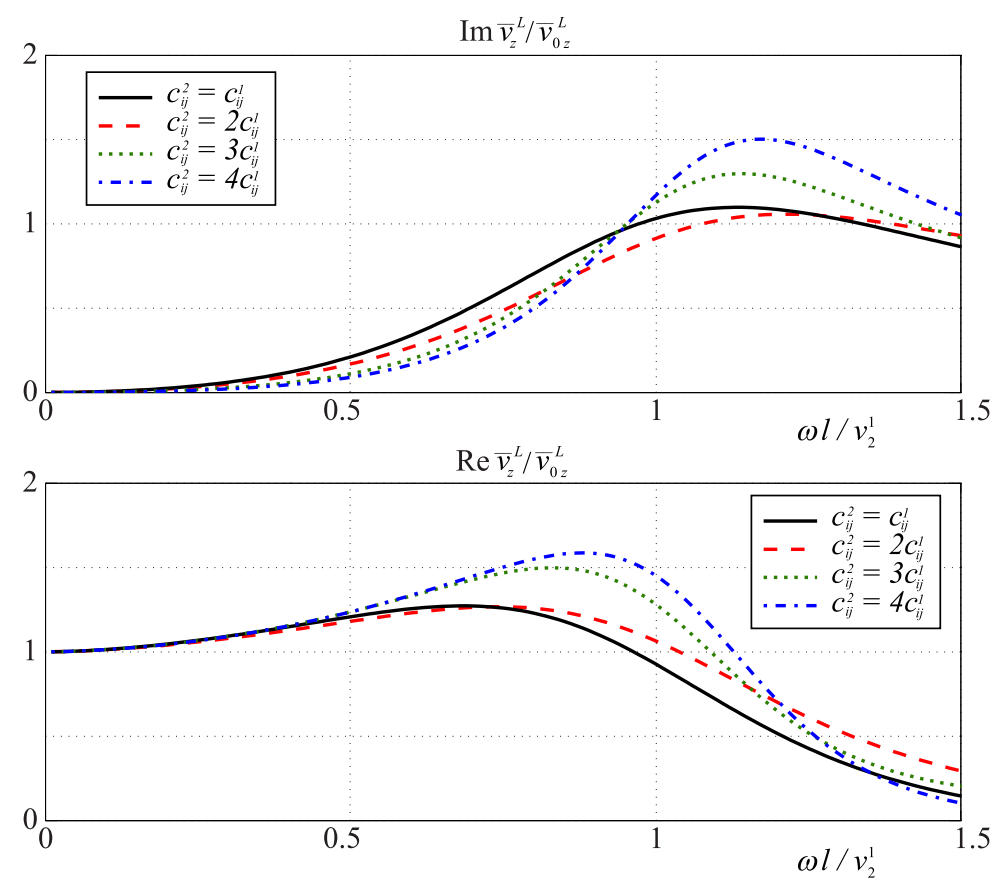

Figure 2: The real and imaginary part of the ratio $\bar{v}_{z}^{L} / \bar{v}_{0 z}^{L}$ between the average value of the exact solution and the low frequency solution of the integral equation.

\section{Random distribution of interface cracks}

Following the scheme used for the SH case [14], consider a plane P or SV wave propagating normally to an interface with a distribution of cracks of the same width $2 l$, see Figure 3 . In this section the situation with a random distribution of cracks is investigated and in the next section this is compared to a periodic distribution of cracks. For a random distribution the assumption of cracks of the same size is not important and the results can easily be generalized to a distribution in size. The crack density parameter $C$ is introduced as the ratio of the cracked part with $N_{c}$ cracks to the total segment of length $x_{0}$ (which is assumed to be large)

$$
C=N_{c} l / x_{0}
$$

The parameter $C$ can be viewed as a damage parameter when the cracking is assumed to be due to interface damage. For a periodic array of cracks the crack density $C$ is evidently simplified to

$$
C=2 l / w,
$$

where $w$ is the distance between the centres of two adjacent cracks.

The total field is written as $\boldsymbol{u}=\boldsymbol{u}^{\text {in }}+\boldsymbol{u}^{\text {sc }}$ as in the Section 2. The incident field $\boldsymbol{u}^{\text {in }}$ is still given by (2), while $\boldsymbol{u}^{\text {sc }}$ is the field scattered by all the cracks. It is assumed that the interaction between the cracks can be neglected [12]. The 

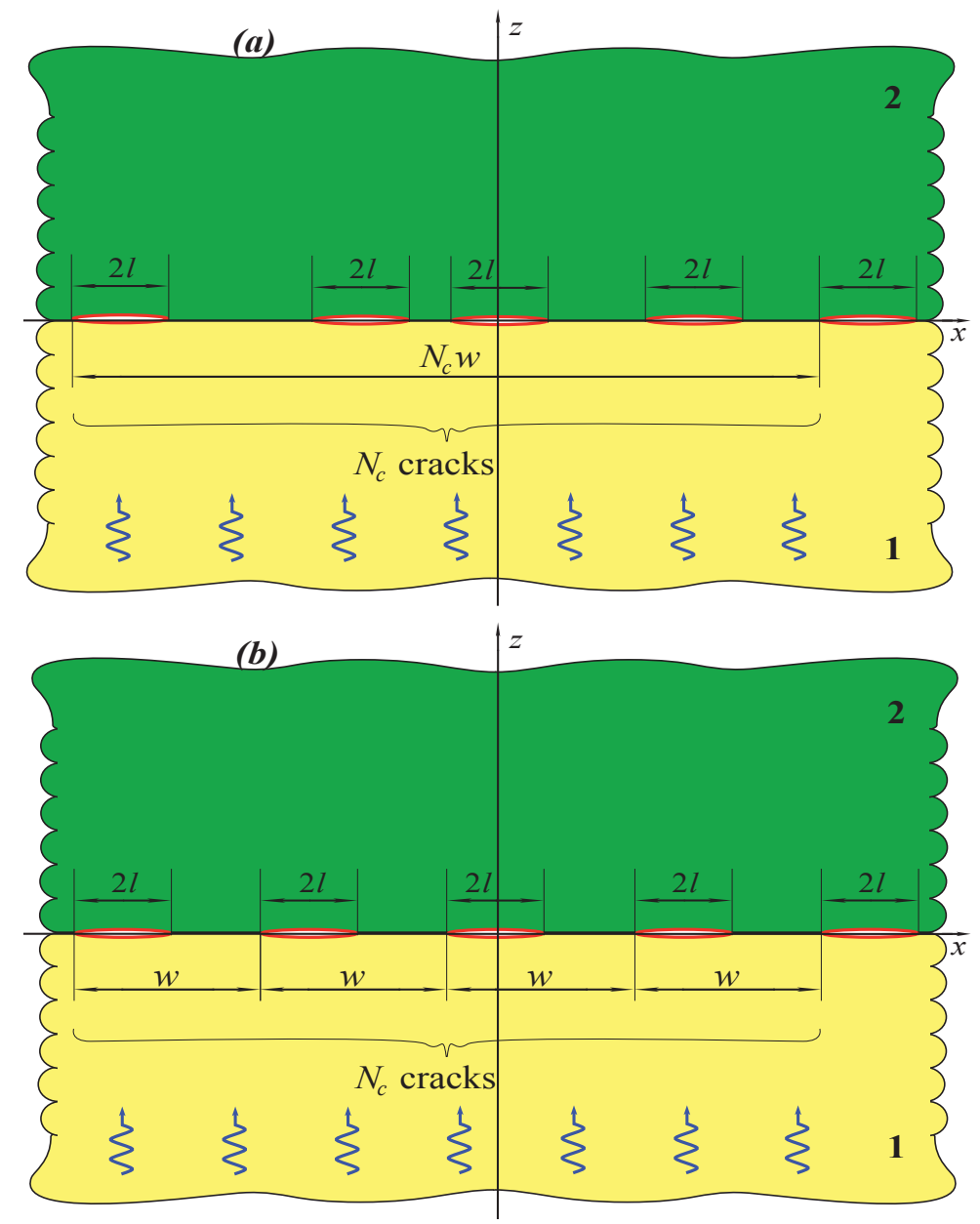

Figure 3: Geometry of the distribution of interface cracks. Random distribution of cracks of the same size (a) and periodic array of cracks (b).

exact scattered field for the random distribution is impossible to determine and is of no interest in fact. Instead the ensemble average of the scattered field is calculated and far from the interface this average field should approximate the total field scattered by the random distribution of cracks. Far away from the interface the ensemble average of the scattered field consists solely of outgoing plane waves propagating in the $\pm z$ direction:

$$
\left\langle\boldsymbol{u}^{\mathrm{sc}}\right\rangle=\boldsymbol{p}^{s}\left\{\begin{array}{cc}
P_{s}^{-} \mathrm{e}^{-\mathrm{i} k_{s}^{1} z}, & z<0 \\
P_{s}^{+} \mathrm{e}^{\mathrm{i} k_{s}^{2} z}, & z>0
\end{array}\right.
$$

The Betty-Rayleigh reciprocal relation to the two elastodynamic states $\boldsymbol{u}^{\text {sc }}$ and $\boldsymbol{u}^{\text {in }}$ is now applied:

$$
\int_{S}\left[\boldsymbol{u}_{i}^{\mathrm{in}} \cdot \boldsymbol{\tau}_{i j}^{\mathrm{sc}}-\boldsymbol{u}_{i}^{\mathrm{sc}} \cdot \boldsymbol{\tau}_{i j}^{\mathrm{in}}\right] n_{j} d S=0 .
$$

The contour $S$ is assumed to be a sum of the rectangular contour $S^{-}$with corners at the points $\left( \pm x_{0}, 0^{-}\right),\left( \pm x_{0},-z_{0}\right)$ and the rectangular contour $S^{+}$with corners at $\left( \pm x_{0}, 0^{+}\right),\left( \pm x_{0}, z_{0}\right)$ which is symmetric to $S^{-}$with respect to the $x$ axis. 
The integrals along the interface then cancel along the uncracked parts and contain the crack-opening displacement along the cracked parts. Taking an ensemble average the other integrals can all be calculated and this gives for the reflection coefficient

$$
P_{s}^{-}=-\frac{1}{2}\left(1-R_{s}^{-}\right) C \boldsymbol{p}^{s} \cdot \overline{\boldsymbol{v}}^{s},
$$

which is expressed in terms of the average value of the crack-opening displacement for a single crack. At low frequencies the asymptotic approximation from the previous section can be used.

The reflection coefficient $P_{s}^{+}$must be determined also. For this purpose the reciprocal relation is used with a plane wave incident from the upper half-space

$$
\boldsymbol{u}^{\mathrm{in}}=\boldsymbol{p}^{s} \begin{cases}T_{s}^{+} \mathrm{e}^{-\mathrm{i} k_{s}^{2} z}, & z<0 \\ \mathrm{e}^{-\mathrm{i} k_{s}^{1} z}+R_{s}^{+} \mathrm{e}^{\mathrm{i} k_{s}^{1} z}, & z>0\end{cases}
$$

where the reflection and transmission coefficients are related to the previous ones:

$$
R_{s}^{+}=-R_{s}^{-}, \quad T_{s}^{+}=1+R_{s}^{+} .
$$

An analogous evaluation of the Betti-Rayleigh relation for the new $\boldsymbol{u}^{\text {in }}$ and the old $\boldsymbol{u}^{\text {sc }}$ (still given by Eq. (8)) gives

$$
P_{s}^{+}=-\frac{1}{2}\left(1+R_{s}^{-}\right) C \boldsymbol{p}^{s} \cdot \overline{\boldsymbol{v}}^{s} .
$$

Subsequently the ensemble average of the total transmission coefficient for the distribution of cracks becomes

$$
\widetilde{T}_{s}=T_{s}^{-}+P_{s}^{+}=T_{s}^{-}\left(1-\frac{1}{2} C \boldsymbol{p}^{s} \cdot \overline{\boldsymbol{v}}^{s}\right) \text {. }
$$

Thus the total transmission by the cracked interface is expressed in terms of the material constants, the length of the cracks and the parameter $C$ describing the density of cracks.

\section{Periodic distribution of interface cracks}

The problem of determining the transmission and reflection coefficients for a periodic distribution of interface cracks can be solved in essentially the same way as for a single crack. The problem with periodic cracks in an otherwise homogenous material, the special case when the half-spaces are of the same material, is considered by Mikata [9], so only a few steps are indicated here. The displacement jump $v$ on the interface is of course the sum of the crack-opening displacements $\boldsymbol{v}_{j}, j=0, \pm 1, \pm 2 \ldots$, on each crack. Thus, instead of the integral equation (4) for a single crack the singular integral equation becomes

$$
\frac{1}{2 \pi} \int_{-\infty}^{\infty} L(\alpha) \sum_{j=-\infty}^{\infty} \boldsymbol{V}_{j}^{s}(\alpha) \mathrm{e}^{\mathrm{i} \alpha x} \mathrm{~d} \alpha=-\mathrm{i} c_{s}^{1} k_{s}^{1}\left(1-R_{s}^{-}\right) \boldsymbol{p}^{s}, \quad|x|<l .
$$

For normal incidence the crack-opening displacements on the cracks are all identical and after a Fourier transform this means

$$
\boldsymbol{V}_{j}^{s}(\alpha)=\boldsymbol{V}_{0}^{s}(\alpha) \exp (\mathrm{i} \alpha w j)
$$


The crack-opening displacement is again expanded in the Chebyshev functions, exactly as for a single crack

$$
\boldsymbol{v}_{0}^{s}=\sum_{k=1}^{\infty} \boldsymbol{\alpha}_{k}^{s} \psi_{k}(x / l)
$$

Projecting also on the Chebyshev functions leads to the discretized integral equation:

$$
\sum_{n=1}^{\infty} \widehat{Q}_{n n^{\prime}}^{s} \boldsymbol{\alpha}_{n^{\prime}}^{s}=-\mathrm{i} K_{s} l \boldsymbol{p}^{s} \delta_{n 1} .
$$

The matrix $\widehat{Q}_{n n^{\prime}}^{s}$ can be evaluated using the following relation reorganizing a sum of delta functions into an exponential series

$$
\sum_{j=-\infty}^{\infty} \mathrm{e}^{\mathrm{i} \beta j}=\sum_{j=-\infty}^{\infty} \delta\left(\frac{\beta}{2 \pi}-j\right)
$$

so that the result is

$$
\widehat{Q}_{n n^{\prime}}^{s}=-\left.\sum_{j=-\infty}^{\infty} L\left(\alpha_{j}\right) \frac{\mathbf{J}_{n}\left(\alpha_{j} l\right) \mathbf{J}_{n^{\prime}}\left(-\alpha_{j} l\right)}{w\left(\alpha_{j} l\right)^{2}}\right|_{\alpha_{j}=2 \pi j / w} .
$$

Once the crack-opening displacement is determined it is straightforward to calculate the transmission and reflection coefficients, see Mikata [9].

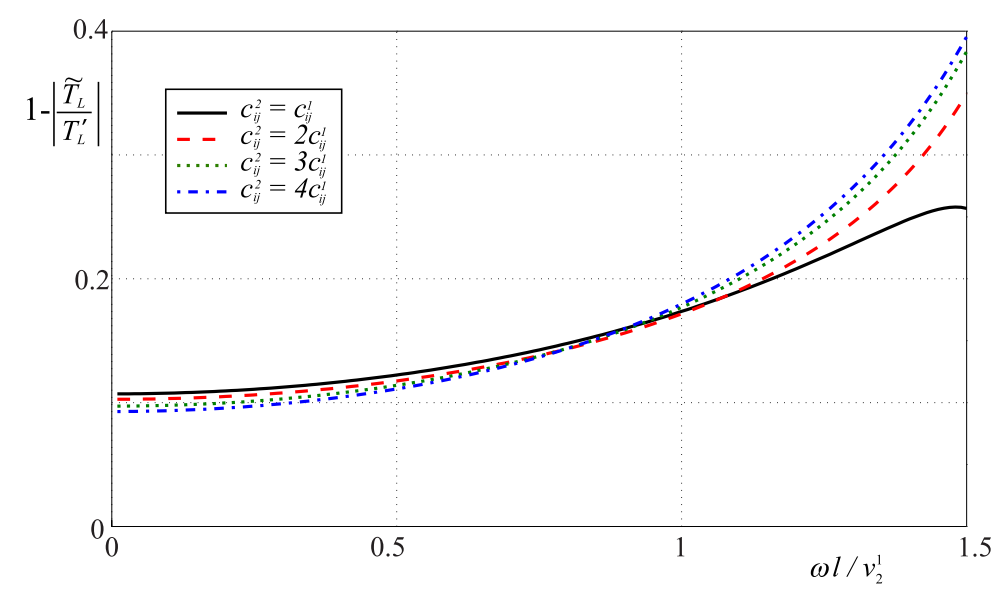

Figure 4: Relative difference $1-\left|T_{L}^{\prime}\right| /\left|\widetilde{T_{L}}\right|$ between the scattered field by periodic array of cracks $T_{L}^{\prime}$ and by random distribution of cracks $\widetilde{T}_{L}$.

The random and periodic distribution of interface cracks can both be seen as models of the situation with interface damage. It is therefore of interest to see how close these are to each other. Figure 4 shows the relative difference between the amplitude transmission coefficients for the random $\widetilde{T}_{L}$ and periodic $T_{L}^{\prime}$ distribution of cracks as a function of frequency. The densities and Poisson's ratios of the half-spaces are equal $\left(\rho^{1}=\rho^{2}, v^{1}=v^{2}=0.3333\right)$ and four different ratios between the elastic constants are considered: $c_{i j}^{2}=B c_{i j}^{2}, B=1,2,3,4$, where $c_{i j}$ is any of the elastic constants. At low frequencies the relative difference is about $10 \%$, so the two distributions give quite similar results. The difference is in fact smaller than could be expected from other uncertainties in the model, such as different crack 
sizes or partially closed cracks. The difference in transmission coefficient of the random and periodic distribution is in accordance with results of Sotiropoulos and Achenbach [12], where statistical and periodic distributions are compared. As the random distribution of cracks leads to simple, explicit expressions, this model is used in the following when the spring boundary conditions are derived.

\section{Spring boundary conditions}

The random distribution of cracks is now transformed into a model with an equivalent spring boundary condition. This boundary condition demands that the stress is continuous while the jump in displacement is proportional to the stress:

$$
\tau^{1}=\tau^{2}=\kappa\left(u^{1}-u^{2}\right)
$$

Here $\kappa$ is a two-by-two matrix, whose elements are determined by a comparison with the transmission coefficients for the random distribution of cracks. In this process a normally incident incoming $\mathrm{P}$ wave is used to determine the normal spring component $\kappa_{22}=\kappa_{L}$ and an S wave to determine the tangential spring component $\kappa_{11}=\kappa_{S}$. The offdiagonal elements can be assumed to vanish as the incoming $\mathrm{P}$ wave which hits the crack gives no scattered $\mathrm{S}$ wave in the forward direction and vice versa.

The incoming wave is still normally incident plane wave from below, exactly as in Section 2:

$$
\boldsymbol{u}^{s}= \begin{cases}\boldsymbol{p}^{s}\left(\mathrm{e}^{\mathrm{i} k_{1}^{1} z}+\widehat{R}_{s} \mathrm{e}^{-\mathrm{i} k_{s}^{1} z}\right), & z<0, \\ \boldsymbol{p}^{s} \widehat{T}_{s} \mathrm{e}^{\mathrm{i} k_{s}^{2} z}, & z>0,\end{cases}
$$

The transmission and reflection coefficients are easily calculated for the spring boundary conditions:

$$
\begin{aligned}
& \widehat{R}_{s}^{-}=\frac{\mathrm{i} c_{s}^{1} k_{s}^{1} c_{s}^{2} k_{s}^{2}+\kappa_{s}\left(c_{s}^{1} k_{s}^{1}-c_{s}^{2} k_{s}^{2}\right)}{\mathrm{i} c_{s}^{1} k_{s}^{1} c_{s}^{2} k_{s}^{2}+\kappa_{s}\left(c_{s}^{1} k_{s}^{1}+c_{s}^{2} k_{s}^{2}\right)}, \\
& \widehat{T}_{s}^{-}=\frac{2 \kappa_{s} c_{s}^{1} k_{s}^{1}}{\mathrm{i} c_{s}^{1} k_{s}^{1} c_{s}^{2} k_{s}^{2}+\kappa_{s}\left(c_{s}^{1} k_{s}^{1}+c_{s}^{2} k_{s}^{2}\right)} .
\end{aligned}
$$

As before $s=1,2$ denotes an incoming $\mathrm{P}$ or $\mathrm{S}$ wave, respectively.

To determine $\kappa_{s}$ the expression for $\widehat{T}_{s}^{-}$should now be put equal to the transmission coefficient for the random distribution of cracks given by Eq. (9). Using also the low frequency approximation for the crack opening displacement Eq. (6) or (7) this gives

$$
\kappa_{s}=\frac{8}{\pi C l \beta_{1}}-\mathrm{i} H_{s}
$$

This equation can be used as is and this leads to a complex spring constant, which leads to energy losses. However, making $\kappa_{s}$ dimensionless by dividing with $c_{s}^{1} k_{s}^{1}$ it is seen that the first term dominates for low frequencies $\left(k_{s}^{1} l\right.$ small), so the last term can be neglected and the final result for the spring constant becomes

$$
\kappa=\frac{8}{\pi C l \beta_{1}}
$$


As the spring constant becomes the same for $s=1,2$, the index $s$ on $\kappa$ is omitted. This means that the spring matrix in Eq. (10) becomes the scalar $\kappa$ in Eq. (12). The spring constant is also frequency independent, and this means that the present spring boundary conditions can be used also in the time domain. However, if the present spring constant is compared with the one for the anti-plane ( $\mathrm{SH}$ ) case as given by Boström and Golub [14], it differs in that the elastic constants enter in another way.

\section{Dispersion properties}

(a) Identical materials

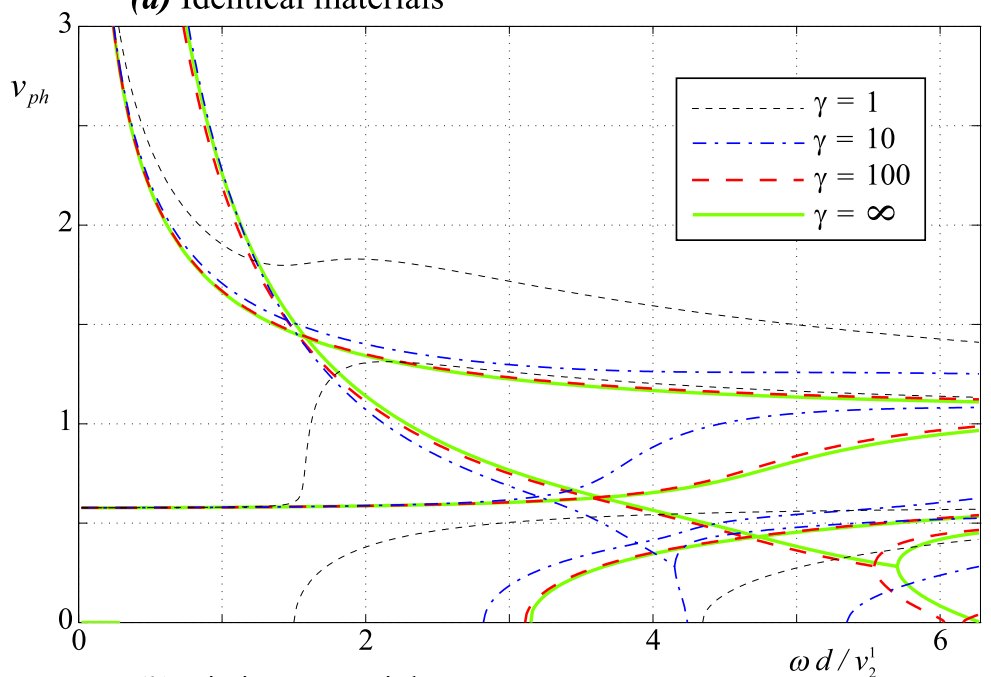

(b) Distinct materials

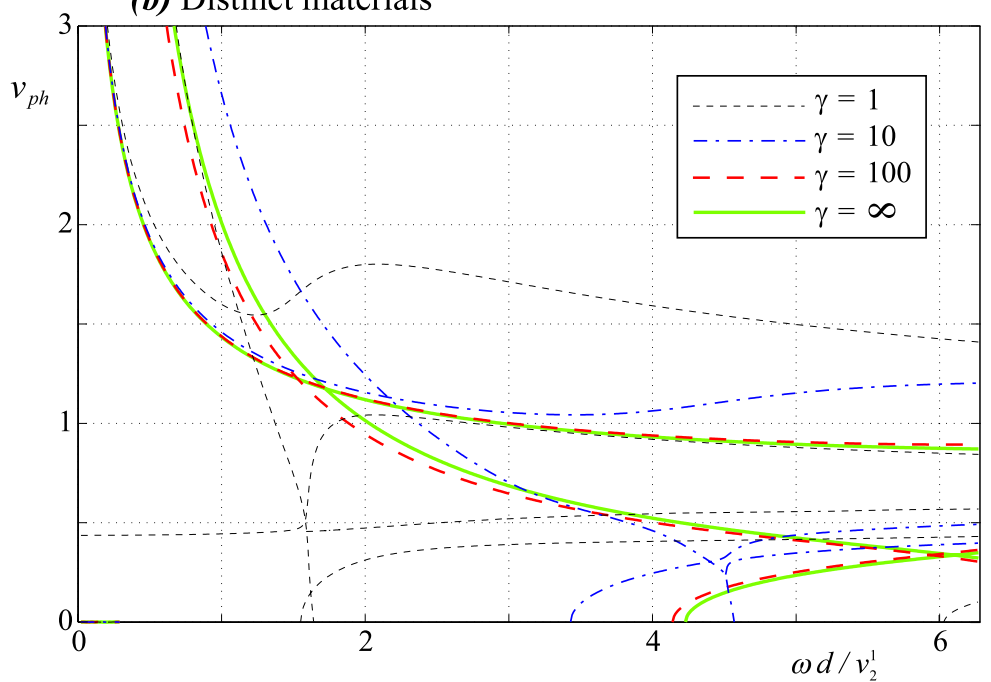

Figure 5: Phase velocities of the first modes for two-layered plate with spring boundary conditions (10): (a) similar materials, (b) distinct materials.

To give an idea of the influence of the spring boundary condition, a simple example with the dispersion relation for a layered plate is now presented. A plate with total thickness $d=d_{1}+d_{2}$ composed of two layers of thicknesses 
$d_{1} / d=0.25$ and $d_{2} / d=0.75$ is considered. The two layers are connected with the spring boundary condition, while the outer surfaces are traction-free. To measure the strength of the interface it is convenient to introduce the dimensionless spring constant $\gamma$ according to:

$$
\gamma=\kappa \frac{\mu^{1} d}{\left(\rho^{1}\right)^{2}} .
$$

Note that this normalization is made so that $\gamma$ is frequency independent. However, it is then necessary to use a length, in this case the plate thickness $d$, to normalize with. This is somewhat unnatural as this length has nothing to do with the interface. It is noted that $\gamma$ must be large for the developments in previous sections to be valid. The spring constant value $\gamma \rightarrow \infty$ corresponds to perfect (welded) contact.

The densities in the two layers are assumed equal and the elastic constants are either equal or those in layer 2 are twice those in layer 1: $c_{i j}^{2}=2 c_{i j}^{1}$, with $c_{i j}^{k}$ any of the elastic constants. The Poisson ratios of the both layers are $v^{1}=v^{2}=0.33333$. The wave number in the direction of propagation is $k$ and the dispersion plots show the dimensionless phase velocity $v_{p h}=1 / k d$ as function of dimensionless frequency $\omega d / v_{2}^{1}$.

Figures $5 \mathrm{a}$ and $\mathrm{b}$ show the dispersion curves for identical and different material properties in the two layers, respectively, for different values of the spring constant. The curves are quite complicated, especially for smaller $\gamma$. The curves for the large value $\gamma=100$ hardly differ from welded contact $(\gamma \rightarrow \infty)$, but already $\gamma=10$ gives relatively large deviations, and $\gamma=1$ even more so, of course. However, the present model may not be valid as a model for interface damage when $\gamma=1$.

\section{Concluding remarks}

The main goal of the present paper is to investigate an interface with damage. This damage is modeled as a random distribution of small cracks of equal size. This model is then transformed into a spring boundary condition and a very simple expression is obtained for the spring constant. The use of this spring boundary condition is illustrated with the dispersion curves for a two-layered plate with interface damage.

While the derivation of the spring stiffness is performed for normal incidence of a plane wave, it is plausible that is a good approximation for any direction of incidence. In the SH case this has been demonstrated by Boström and Kvasha [19] where the dispersion relation (where non-normal directions are essential) in a layered plate with damage modeled by spring boundary conditions (derived as here) or a periodic array of interface cracks (solved exactly as here) are compared with a good correspondence.

The present methodology can be extended in several directions. Both the 3D case and anisotropy are of interest to make the spring boundary conditions for damage accessible to a wider range of problems. To investigate the importance and detectability of damage in various situations, specific problems should be studied. This may be an interface with only partial damage, damage in a layered plate, etc. 


\section{Acknowledgments}

The authors are grateful to Professor E.V. Glushkov and N.V. Glushkova for the help and valuable advices. The work is supported by the Ministry of Education and Science of Russian Federation (project 2.1.1/1231).

\section{References}

[1] S. Nakagawa, K.T. Nihei and L.R. Myer, "Plane wave solution for elastic wave scattering by a heterogeneous fracture", Journal of the Acoustical Society of America 115, 2761-2772 (2004).

[2] C. Pecorari, "Spring boundary model for a partially closed crack", International Journal of Engineering Sciences 46, 182-188 (2008).

[3] R. Leiderman, A.M.B. Barbone and P.E. Braga, "Scattering of ultrasonic waves by defective adhesion interfaces in submerged laminated plates" Journal of the Acoustical Society of America 118, 2154-2166 (2005).

[4] J.M. Baik and R.B. Thompson, "Ultrasonic scattering from imperfect interfaces: a quasi-static model", Journal of Nondestructive Evaluation 4, 177-196 (1984).

[5] S.I. Rokhlin and Y.J. Wang, "Analysis of boundary conditions for elastic wave interaction with an interface between two solids", Journal of the Acoustical Society of America 89, 503-515 (1991).

[6] S.I. Rokhlin and W. Huang, "Ultrasonic wave interaction with a thin layer between two anisotropic solids", Journal of the Acoustical Society of America 92, 1729-1742 (1992).

[7] Y.C. Angel and J.D. Achenbach, "Reflection and transmission of elastic waves by a periodic array of cracks", Journal of Applied Mechanics 52, 33-41 (1985).

[8] Y. Mikata and J. D. Achenbach, "Interaction of harmonic waves with a periodic array of inclined cracks", Wave Motion 10, 59-72 (1988).

[9] Y. Mikata, "Reflection and transmission by a periodoc array of coplanar cracks: normal and oblique incidence", Journal of Applied Mechanics 60, 911-919 (1993).

[10] J.D. Achenbach and Ch. Zhang, "Reflection and transmission of ultrasound by a region of damaged material", Journal of Nondestructive Evaluation 9, 71-79 (1990).

[11] J.D. Achenbach, I.M. Kitahara, Y. Mikata and D.A. Sotiropoulos, "Reflection and transmission of plane waves by a layer of compact inhomogeneities", Pure and Applied Geophysics 128, 101-118 (1988).

[12] D.A. Sotiropoulos and J.D. Achenbach, "Ultrasonic reflection by a planar distribution of cracks", Journal of Nondestructive Evaluation 7 , 123-129 (1988).

[13] A. Boström and G.R. Wickham, "On the boundary conditions for ultrasonic transmission by partially closed cracks", Journal of Nondestructive Evaluation 10, 139-149 (1991).

[14] A. Boström and M. Golub, "Elastic SH wave propagation in a layered anisotropic plate with interface damage modelled by spring boundary conditions", Quarterly Journal of Mechanics and Applied Mathematics 62, 39-52 (2009).

[15] A. Boström, "Review of hypersingular integral equation method for crack scattering and application to modeling of ultrasonic nondestructive evaluation", Applied Mechanics Reviews 56, 383-405 (2003).

[16] E. Glushkov and N. Glushkova, "On the efficient implementation of the integral equation method in elastodynamics", Journal of Computational Acoustics 9, 889-898 (2001).

[17] V.A. Babeshko, E.V. Glushkov, J.F. Zinchenko, Dynamics of inhomogeneous linear-elastic media, Nauka, Moscow, 1989 (in Russian).

[18] K.N. Srivastava, R.M. Palaiya and O.P. Gupta, "Interaction of longitudinal wave with a penny-shaped crack at the interface of two bonded dissimilar elastic solids-II", International Journal of Fracture 15, 591-599 (1979).

[19] A. Boström and O.V. Kvasha, "Elastic SH wave propagation in a layered anisotropic plate with periodic interface cracks: exact versus spring boundary conditions", Journal of Materials and Structures, 5(1), 67-78 (2010). 